DOI: $10.22481 /$ recuesb.v8i14.7844

\title{
FALE COM OBSTETRAS - CAMPINA GRANDE: RELATO DE EXPERIÊNCIA DE UM PROJETO INTERPROFISSIONAL
}

\section{TALK TO OBSTETRICIANS - CAMPINA GRANDE: EXPERIENCE REPORT OF AN INTERPROFESSIONAL PROJECT}

\author{
Arthur Ferreira Cerqueira Amorim ${ }^{1}$ \\ Luanna Cristiny Domingos Nobre ${ }^{2}$ \\ Beatriz Berenguer de Souza Freitas ${ }^{3}$ \\ Maria Eduarda Gurgel Medeiros ${ }^{4}$ \\ Katarina Venâncio Antunes Romeu Ramos ${ }^{5}$ \\ Melania Maria Ramos de Amorim ${ }^{6}$
}

\begin{abstract}
Resumo: Introdução: em dezembro de 2019, foi identificada uma nova variação do coronavírus, altamente transmissível, denominado SARS-CoV-2, causando a doença posteriormente nomeada como COVID-19. Apesar de muitos estudos já publicados em tão curto espaço de tempo sobre a doença, ainda há muitas dúvidas em relação a assuntos mais específicos, como a gestação. Objetivo: o objetivo deste relato de experiência é descrever o modelo de implementação do programa "Fale com Obstetras", destacando as práticas realizadas por discentes de medicina durante sua execução e apresentar os resultados iniciais, com enfoque na promoção à saúde de gestantes e puérperas. Métodos: o método de execução do projeto se deu, sobretudo, através de dois aplicativos gratuitos para smartphones: o WhatsApp ${ }^{\circledR}$, através do qual foram realizadas as orientações; e o Instagram ${ }^{\circledR}$, onde foram disponibilizados os conteúdos informativos. Resultados: até o presente momento, foram atendidas 127 pacientes,
\end{abstract}

\footnotetext{
${ }^{1}$ Graduando em Medicina, Universidade Federal de Campina Grande (UFCG), Campina Grande, Paraíba, Brasil. Orcid: https://orcid.org/0000-0003-4210-6180. E-mail: arthuramorim.ufcg@gmail.com

${ }^{2}$ Graduanda em Medicina, Universidade Federal de Campina Grande, (UFCG), Campina Grande, Paraíba, Brasil. Orcid: https://orcid.org/0000-0002-6049-3850 E-mail: luannacristiny13@gmail.com

${ }^{3}$ Graduanda em Medicina, Universidade Federal de Campina Grande, (UFCG), Campina Grande, Paraíba, Brasil. Orcid: https://orcid.org/0000-0003-3319-1061 E-mail: beatrizberenguersf@gmail.com

${ }^{4}$ Graduanda em Medicina, Universidade Federal de Campina Grande, (UFCG), Campina Grande, Paraíba, Brasil. Orcid: https://orcid.org/0000-0002-1888-5930 E-mail: mariaeduardagurgel@outlook.com

${ }^{5}$ Graduanda em Medicina, Universidade Federal de Campina Grande, (UFCG), Campina Grande, Paraíba, Brasil. Orcid: https://orcid.org/0000-0003-2758-6438. E-mail: katarinapicui@gmail.com

${ }^{6}$ Médica Ginecologista e Obstetra; Doutora em Tocoginecologia. Professora da Universidade Federal de Campina Grande, (UFCG), Campina Grande, Paraíba, Brasil. Orcid: https://orcid.org/0000-0003-1047-2514. E-mail: profmelania.amorim@gmail.com
} 
com faixa etária média de 27,5 anos, com queixas e dúvidas diversas, das quais 78 tiveram suas dúvidas sanadas através dos aplicativos, sendo as outras 49 orientadas a procurar os serviços de saúde. Discussão: é possível observar que o projeto foi capaz não somente de diminuir as aflições e consequências geradas pela pandemia, como a extrema ansiedade e o isolamento social, mas também pôde sintetizar as principais queixas das pacientes, permitindo que seu atendimento fosse direcionado corretamente. Conclusão: a pandemia da COVID-19 trouxe a necessidade de se buscar formas de atendimento seguro para a população em geral, sendo a implementação de um serviço de teleorientação para gestantes uma dessas formas.

Palavras-chave: COVID-19. Teleorientação. Gestação. Puerpério.

Abstract: Introduction: In December 2019, a new highly transmissible variation of Coronavirus was identified, named SARS-CoV-2, which is responsible for causing COVID-19. Although many studies have already been published about this disease, there are still many doubts about some specific aspects, such as its effects in pregnancy. Objective: This experience report mainly objective is to describe the implementation model of the "Fale com Obstetras" program (Talk to Obstetricians, in English), highlighting how medical students proceeded during it's execution and present the initial results, focusing on promoting health support to pregnant and puerperal women. Methods: the execution method of the present project was mostly through two smartphone apps: WhatsApp $\mathbb{R} \square$, which was the device where the patients received orientation; and Instagram $\mathbb{\circledR} \square$, where informative content was made available. Results: until the present moment, 127 patients were attended, with an average age of 27.5 years and diverse complaints and doubts, which 78 had their questions answered by online attending and 49 others were asked to attend an in-person appointment in public health system. Discussion: It is possible to observe that the project was able to not only minimize the afflictions and consequences caused by the pandemic, as extreme anxiety and social isolation, but also it could solve the main complaints, allowing that patients' attending were correctly directed. Conclusion: COVID-19 pandemic brought the necessity to search for ways to attend safely the general population, such as the implementation of an online service for pregnant women.

Keywords: COVID-19. Tele-orientation. Pregnancy. Postpartum period.

\section{Introdução}

Em dezembro de 2019, foi identificada uma nova variação do coronavírus em Wuhan, China: o SARS-CoV-2 (síndrome respiratória aguda de coronavírus 2). Essa manifestação, denominada COVID-19 (do inglês coronavirus disease) pela Organização Mundial da Saúde 
(OMS), que é altamente transmissível, sendo letal em alguns casos, apresenta sintomas predominantemente respiratórios e representa atualmente a causa de uma das maiores crises sanitárias mundiais, tendo sido declarada como pandemia pela OMS, em 11 de março de 2020. Apesar de ter sido gerado um conhecimento quase que instantâneo acerca dessa infecção e já existirem inúmeros estudos relacionados aos diferentes aspectos da infecção, surgiram muitas dúvidas sobre os possíveis efeitos da COVID-19 na gravidez e da gravidez sobre o curso da COVID-19 e só, recentemente, têm sido publicados estudos que evidenciam maior risco de complicações durante a infecção por COVID-19 na gravidez e no puerpério, bem como relatos de efeitos adversos na gravidez como, por exemplo, restrição de crescimento, óbito fetal e síndrome pré-eclâmpsia-like, além de síndrome HELLP e hemorragia pós-parto (MENEZES et al., 2020).

Em âmbito global, conforme os dados da OMS, já foram identificados, até o dia 5 de dezembro de 2020, 65.007.974 de casos confirmados e 1.507.018 mortes. A todo momento, há mudanças, sejam elas instantâneas ou graduais, em relação à epidemiologia global da doença, como no padrão de aumento no número de casos e na localização dos "epicentros". China e Itália, por exemplo, foram consideradas epicentro no início da pandemia, no entanto, atualmente, o foco da doença atinge os Estados Unidos, Índia e o Brasil, os três países com mais mortes registradas por COVID-19 no mundo (WHO et al., 2020).

Em âmbito nacional, até o dia 5 de dezembro de 2020, foram confirmados 6.533.968 casos de COVID-19, com 175.964 óbitos. No Nordeste, segunda região mais afetada no País, foram confirmados 1.666.151 casos, destes, 416.734 na Bahia, 303.868 no Ceará e 148.003 na Paraíba (MINISTÉRIO DA SAÚDE, 2020).

Há muita preocupação com os efeitos da pandemia na saúde materna e perinatal, não somente pelos diretos, mas também pelos efeitos indiretos. Por exemplo, já se demonstrou que vários serviços reduziram, cancelaram ou postergaram as consultas pré-natais. A recente pesquisa nacional do Instituto Patrícia Galvão com o Instituto Locomotiva, apoiada pela ONU Mulheres, demonstrou que uma em cada três mulheres grávidas teve acesso diminuído a consultas e exames pré-natais durante a pandemia no Brasil (IPG, 2020). Isso gera

$$
\begin{array}{l|l}
\multirow{2}{*}{\text { REVISTA }} & \text { EXTENSÃO \& CIDADANIA } \\
\cline { 2 - 3 } & \text { v. 8, n. 14, p. 399-413, jul./dez. 2020. ISSN 2319-0566 }
\end{array}
$$


desinformação, medos, anseios e preocupações entre as grávidas e também pode acarretar desfechos gestacionais adversos. Estudos em outros países já demonstraram aumento da frequência de óbitos fetais, associados à carência da assistência pré-natal.

Quando analisada a epidemiologia de pandemias anteriores é possível inferir que gestantes possuem maior risco de doenças graves e morte devido a infecções virais (LEVY et al., 2008), em decorrência das alterações anatômicas e fisiológicas no sistema cardiorrespiratório, além das alterações imunológicas. Mesmo que os estudos iniciais não apontassem riscos aumentados, todos os estudos mais recentes demonstraram que gestantes têm maior risco de complicações da COVID-19, de internação, internação em UTI e precisar de ventilação mecânica (AMORIM et al., 2020). Além disso, os estudos brasileiros do grupo “Brazilian Group for Studies of COVID-19 e gravidez" têm demonstrado um elevado número de mortes maternas por COVID-19 no país, superior a todas as mortes por COVID-19 no restante do mundo.

Diante do impacto global da pandemia, medidas sociais foram recomendadas por especialistas como a principal forma de impedir a propagação do vírus e um possível colapso dos serviços de saúde (ANDERSON et al., 2020), partindo de estratégias básicas como o isolamento social até complexas, como a instauração de barreiras sanitárias (DEISY, 2020). No entanto, essas medidas, ainda que necessárias, deflagraram outros tipos de prejuízo à saúde da mulher, como a interrupção de serviços essenciais, como pré e pós-natais, contracepção e aborto seguro (TANG et al., 2020).

Nesse sentido, apesar de as diretrizes de saúde nacionais e internacionais protegerem o acesso de gestantes aos serviços essenciais, estabelecendo a obrigatoriedade de se manter a assistência pré-natal, torna-se claro que a necessária readequação da realidade pode ter contribuído para a interrupção de determinados serviços, colocando em xeque a saúde, já relativamente mais frágil, dessa parcela da população. Dessa forma, o projeto "Fale com Obstetras - Campina Grande" (FCOO-CG) surgiu como uma ferramenta voluntária que objetivou auxiliar as mulheres grávidas da região através de teleorientações.

Entre seus principais objetivos, destacam-se a contribuição para o isolamento social;

$$
\begin{array}{l|l}
\multirow{2}{*}{\text { REVISTA }} & \text { EXTENSÃO \& CIDADANIA } \\
\cline { 2 - 3 } & \text { v. 8, n. 14, p. 399-413, jul./dez. 2020. ISSN 2319-0566 }
\end{array}
$$


redução da exposição desnecessária nos serviços de saúde; desenvolvimento de atividades educacionais nas redes sociais; amenização da aflição e ansiedade e, também, esclarecimento de dúvidas dessa parcela da população destacando, principalmente, o respeito às gestantes e às puérperas participantes, às condutas médicas e às recomendações da OMS.

A pertinência deste trabalho se expressa através de todas as atividades praticadas para a consolidação das ações de promoção à saúde; educacionais e pela experiência interprofissional ofertada aos alunos.

O objetivo deste relato de experiência é descrever o modelo de implementação do programa "Fale com Obstetras", destacando as práticas realizadas por discentes do curso de Medicina durante sua execução e apresentar os resultados iniciais com enfoque na promoção à saúde de gestantes e puérperas.

\section{Método}

O projeto teve como base a proposta "Fale com a Parteira", iniciado por enfermeiras de Pernambuco, que tomou, posteriormente, proporções nacionais (TEIXEIRA, 2020). Propostas semelhantes foram idealizadas e colocadas em prática em várias partes do mundo, como as ações do Departamento de Odontologia (DOD), da Universidade Estadual de Maringá (UEM), que buscou incentivar o autocuidado, além de levar informações relacionadas à prevenção, às causas, ao desenvolvimento e ao tratamentos de doenças bucais (DORNA, 2020) e das Associações Colombianas do Sistema Digestivo, que afirmam que a teleorientação é uma ferramenta valiosa para o paciente, sendo ambas as propostas implementadas após o início da pandemia. Tanto os Conselhos de Medicina, de Enfermagem e de Psicologia como diversos outros da área de saúde permitiram e incentivaram a prática do teleatendimento e teleorientação (MENDES, 2020).

Foi executado através de dois aplicativos gratuitos para smartphones, o WhatsApp ${ }^{\circledR}$ e o Instagram $^{\circledR}$. Para execução, o projeto foi dividido em três grandes grupos principais: capacitação, interação e atendimento. 
A capacitação ocorreu durante o primeiro mês do projeto, semanalmente, foram realizadas duas reuniões para desenvolver maior conhecimento teórico-prático sobre o assunto. A primeira reunião foi realizada com a coordenadora e a segunda entre os alunos e todas foram feitas através de plataformas online para manter o distanciamento social.

A interação se deu através de um perfil no aplicativo Instagram $^{\circledR}$, foram dispostos, através de textos semanais, lives e vídeos quinzenais e quizzes mensais, conteúdos específicos - informativos sobre gravidez, parto e puerpério, preparados pelos estudantes de Medicina sob supervisão das orientadoras. Todas as publicações foram abertas para perguntas e respostas na própria plataforma e permaneceram disponíveis para que fossem acessadas por qualquer pessoa interessada sempre que necessário. Além do atendimento, foram enviadas, também pelo WhatsApp $^{\circledR}$, para as pacientes que desejaram, todas as publicações feitas no Instagram $^{\circledR}$, através de uma lista de transmissão.

$\mathrm{O}$ atendimento foi realizado através do aplicativo WhatsApp ${ }^{\circledR}$, diariamente, entre os horários $8 \mathrm{~h}-12 \mathrm{~h}$ e $14 \mathrm{~h}-18 \mathrm{~h}$, por médicos(as), enfermeiros(as) e psicólogos(as) voluntários(as) e seguiu uma sequência pré-estabelecida de etapas, regidas através de mensagens padronizadas. O staff do projeto foi dividido em dois grupos: reguladores e atendentes.

O grupo da regulação, composto pelos integrantes do projeto, teve como principal função a organização do grupo e do fluxo de atendimentos. Desta forma, coube a eles observar se todas as mulheres que solicitaram ajudam foram atendidas e, em caso negativo, comunicar ao plantonista; orientar as pacientes já atendidas, ao final do plantão, para que saíssem do grupo, evitando superlotação; impedir que conversas paralelas ou propagandas fossem enviadas no grupo, para evitar possível perda de dados; administrar o perfil do Instagram $^{\circledR}$; alimentar a lista de transmissão e manter atualizada a planilha dos atendimentos.

O grupo de atendimento, composto por médicos(as) e enfermeiros(as) voluntários(as), foi encarregado de entrar em contato com a paciente que solicitar ajuda no grupo, respeitando a ordem das solicitações; enviar o termo de consentimento informado para teleorientação de medicina e enfermagem; realizar o atendimento; encaminhar a paciente para atendimento

$$
\begin{array}{l|l}
\multirow{2}{*}{\text { REVISTA }} & \text { EXTENSÃO \& CIDADANIA } \\
\cline { 2 - 3 } & \text { v. 8, n. 14, p. 399-413, jul./dez. 2020. ISSN 2319-0566 }
\end{array}
$$


psicológico, caso ache necessário ou seja solicitado; repassar os dados do atendimento para o regulador responsável pelo horário.

Durante a realização do projeto, nenhuma participante teve qualquer custo adicional, visto que foi realizado remotamente, sendo necessário apenas que tivessem acesso à internet e um smartphone com acesso ao aplicativo WhatsApp ${ }^{\circledR}$. Os critérios de inclusão adotados foram: ser gestante ou puérpera; entrar voluntariamente no grupo de WhatsApp ${ }^{\circledR}$ do projeto, cujo link foi disponível na página do Instagram ${ }^{\circledR}$ "FCOOCG”; e ser residente de Campina Grande/PB ou de um dos municípios cujo atendimento é referenciado para Campina Grande (atualmente 127 municípios pactuados).

Foi criada uma planilha para coleta de dados com as variáveis de análise: idade; idade gestacional; principais dúvidas e/ou queixas; orientações realizadas; e necessidade de encaminhamento para ambientes físicos de atendimento.

Os dados pertinentes foram coletados durante o atendimento remoto da paciente, através de entrevista semiestruturada, utilizando-se um formulário padrão, pré-codificado para entrada de dados em computador. A coleta dos dados foi feita pelos profissionais responsáveis pela teleorientação das pacientes.

A digitação dos formulários foi realizada em uma planilha do software Excel® 2011, com verificação quinzenal realizada pela orientadora. Todas as participantes assinaram Termo de Consentimento Livre e Esclarecido (TCLE).

A análise estatística foi realizada utilizando-se o mesmo programa Excel@ pelos estudantes supervisionados por sua Orientadora. Foram obtidas tabelas de distribuição de frequência e construídos gráficos para variáveis categóricas e calculadas medidas de tendência central e de dispersão para as variáveis numéricas.

\section{Resultados}

Foram atendidas, no total, 127 pacientes, a faixa etária dessas mulheres variava entre 16 e 43 anos, sendo a idade média 27,5 anos e a mediana de 30 anos. 
A idade gestacional dessas pacientes também variava: mulheres que possuíam desde 7 até 40 semanas de gestação, na iminência de parir. A média da idade gestacional entre essas pacientes era de 21,4 semanas e a mediana era de 30,5 semanas. Também foram atendidas puérperas em momentos distintos do pós-parto.

Dentre os casos atendidos, 78 dúvidas foram sanadas apenas com o atendimento online, sem que houvesse encaminhamento da paciente para o serviço presencial, o que corresponde a aproximadamente $62 \%$ do total. Além disso, 49 pacientes foram encaminhadas para um serviço público especializado de saúde - seja a Unidade Básica de Saúde (UBS), onde a gestante realiza o pré-natal, seja o Instituto de Saúde Elpídio de Almeida (ISEA), em caso de gestações de alto risco, ou até mesmo uma Unidade de Pronto Atendimento (UPA) ou um hospital em casos de emergência - o que representa aproximadamente $38 \%$ do total. Todos esses dados estão resumidos nas tabelas 1 e 2 a seguir:

Tabela 1 - Análise quantitativa dos atendimentos, segundo resolução das queixas. Campina Grande, Paraíba, Brasil, 2020. ( $\mathrm{N}=127)$

\begin{tabular}{c|c|c|c}
\hline & Total de queixas solucionadas & Total de encaminhamentos & Total de pacientes atendidas \\
\hline Resultados & $78(62 \%)$ & $49(38 \%)$ & $127(100 \%)$ \\
\hline Fonte: Projeto FCOO-CG.
\end{tabular}

Tabela 2 - Análise do perfil biológico das pacientes atendidas, segundo idade biológica e idade gestacional. Campina Grande, Paraíba, Brasil, 2020. ( $\mathrm{N}=127)$

\begin{tabular}{c|c|c|c|c}
\hline & $\begin{array}{c}\text { Média de idade das } \\
\text { pacientes }\end{array}$ & $\begin{array}{c}\text { Mediana da idade das } \\
\text { pacientes }\end{array}$ & $\begin{array}{c}\text { Média da idade } \\
\text { gestacional das } \\
\text { pacientes }\end{array}$ & $\begin{array}{c}\text { Mediana da idade } \\
\text { gestacional das } \\
\text { pacientes }\end{array}$ \\
\hline Resultados & 27,5 anos & 30 anos & 21,4 semanas & 30,5 semanas \\
\hline
\end{tabular}

Fonte: Projeto FCOOG-CG. 
Ademais, foram computadas as principais queixas relatadas pelas pacientes, no intuito de avaliar as dúvidas das gestantes durante o período gestacional (Figura 1). Percebeu-se, assim, que a maior parte dos registros estava relacionada às dores no geral: em baixo ventre, lombares e, bem expressivamente, cefaleias. Apesar de, muitas vezes, esse sintoma não se apresentar de forma isolada, era uma queixa bastante comum.

A segunda manifestação mais presente era pressão alta e, na maioria das vezes, apresentava-se associada ao sangramento e, especialmente, com sinais e sintomas sugestivos de pré-eclâmpsia com critérios de gravidade. Outro distúrbio muito comum era diabetes gestacional, que também estava associado à hipertensão em alguns casos.

É válido ressaltar também que, dentre os 127 atendimentos, 6 mulheres testaram positivo para COVID e outras 4 possuíam sintomas de síndromes gripais, enquadrando-se como casos suspeitos para a doença. Grande parte dessas pacientes tinha dúvida sobre serviços públicos especializados para atendimento de gestantes na mesma situação em que elas se encontravam, evidenciando a necessidade da existência desses locais. Todas essas pacientes foram orientadas a procurar o serviço de referência. Essas informações estão resumidas no Gráfico 1 a seguir (Figura 1):

Figura 1 - Gráfico das queixas relatadas, segundo as principais ocorrências. Campina Grande, Paraíba, Brasil, 2020. (N=145)

Outros
Dor em Baixo Ventre
Corrimento
Parto
Pressão arterial
Cefaleia
COVID-19

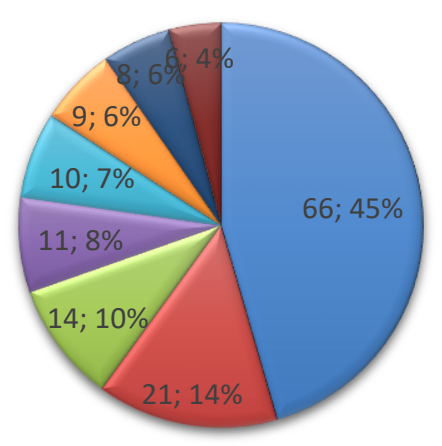

Fonte: Projeto FCOOG-CG.

\begin{tabular}{l|ll}
\multirow{2}{*}{ REVISTA } & EXTENSÃO \& CIDADANIA \\
\cline { 2 - 3 } & v. 8, n. 14, p. 399-413, jul./dez. 2020. & ISSN 2319-0566
\end{tabular} 


\section{Discussão}

Até o presente momento, o Projeto "Fale com Obstetras - CG" atendeu 127 gestantes e conseguiu elucidar muitas dúvidas comuns e orientar atendimento presencial em $38 \%$ dos casos, mas também evitar que gestantes sem indicação fossem desnecessariamente aos serviços de saúde, expondo-se assim ao risco de contrair COVID-19. As principais queixas foram relacionadas com dores diversas, incluindo dores em baixo ventre e cefaleia, mas outras intercorrências obstétricas foram encontradas e abordadas pela equipe, incluindo casos suspeitos de COVID-19 e queixas sugestivas de pré-eclâmpsia com critérios de gravidade, demandando pronta referência ao serviço de saúde

A COVID-19, declarada como pandemia pela OMS em 11 de março de 2020, é uma doença altamente transmissível, podendo ser letal. Com o impacto global gerado pelo novo coronavírus, muitas medidas de restrição foram devidamente adotadas, sendo algumas mais gerais - como o uso da máscara durante todo o tempo em que estiver fora de casa - e outras mais específicas - como a prática de lockdown adotada em algumas regiões (DEISY et al., 2020). Com essas medidas, um dos principais objetivos foi reduzir aglomerações em hospitais públicos, por exemplo, e, consequentemente, evitar colapsos nos serviços de saúde (ROSENBERG et al., 2020).

No que se refere às gestantes, sabe-se que elas foram incluídas como grupo de risco no Brasil desde abril/2020, mas ainda existem inúmeras incógnitas que dificultam um amplo entendimento do mecanismo de ação do vírus nesse grupo populacional (RCOG, 2020). Um estudo publicado pelo CDC dos Estados Unidos comparou a necessidade de internação entre gestantes e não gestantes que contraíram a COVID-19. Concluiu-se que as gestantes possuíam não só maior risco de internação, necessidade de ventilação mecânica e UTI, como também evidenciou maior risco de mortalidade em gestantes (ZAMBRANO, 2020).

O projeto "Fale com Obstetras - Campina Grande" teve como principal objetivo reduzir o atendimento presencial de grávidas e puérperas, promovendo, então, o teleatendimento e protegendo essas mulheres evitando, em muitos casos, consultas presenciais que poderiam

$$
\begin{array}{l|l}
\multirow{2}{*}{\text { REVISTA }} & \text { EXTENSÃO \& CIDADANIA } \\
\cline { 2 - 3 } & \text { v. 8, n. 14, p. 399-413, jul./dez. 2020. ISSN 2319-0566 }
\end{array}
$$


expô-las a locais insalubres, com alta carga viral decorrente da aglomeração em áreas com elevada concentração de indivíduos infectados (LIU et al., 2020).

É importante ressaltar que estudos realizados no início da pandemia não relacionaram as gestantes como grupo de risco para doença grave e mortalidade (ROBERTON et al., 2020) Todavia, pesquisas atuais conseguiram observar que gestantes apresentavam um maior risco de morte, quando comparadas a não gestantes, no que concerne à COVID-19 (TAN et al., 2020). Vale salientar, ainda, que alterações fisiológicas em diversos sistemas de mulheres grávidas estão ligadas ao alto risco ao qual esse grupo está submetido no que se refere às doenças virais (ROBERTON et al., 2020) e que o Brasil concentra o maior número de mortes maternas por COVID-19 no mundo (TAKEMOTO et al., 2020).

O projeto trouxe várias contribuições para as gestantes e puérperas, principalmente no tocante ao atendimento seguro. Ademais, houve a disseminação de informações, embasadas cientificamente e supervisionadas pelas coordenadoras, relacionadas à gestação, ao parto e ao puerpério pelo perfil do projeto no aplicativo Instagram ${ }^{\circledR}$, por meio de textos, lives, vídeos e quizzes. Os conteúdos apresentados no Instagram ${ }^{\circledR}$ foram encaminhados às pacientes que optaram por participar de uma lista de transmissão no aplicativo Whatsapp®.

Nesse contexto, a situação de pandemia pode proporcionar em muitas mulheres grávidas sensações que envolvem medo e ansiedade acerca das possíveis consequências do vírus e da redução dos acompanhamentos médicos durante a gestação. Nesse sentido, o projeto proporcionou, por meio de atividades educativas nas redes sociais, um engajamento coletivo com informes educativos, sendo, portanto, de viés elucidativo.

No que refere aos estudantes de Medicina, coube a coleta de dados, a digitação e organização em planilhas, além de sua atuação como reguladores do projeto. Esse processo proporcionou um contato direto entre os estudantes e os relatos das dificuldades enfrentadas por grávidas e puérperas no período de pandemia, tornando-se uma situação de grande aprendizado. A observação, por exemplo, das queixas obtidas e dos comandos dados pelos profissionais da saúde, os quais estavam atuando no projeto, foi de suma importância para o contato, mesmo que a distância entre os discentes, principalmente os do ciclo básico do curso

$$
\begin{array}{l|l}
\multirow{2}{*}{\text { REVISTA }} & \text { EXTENSÃO \& CIDADANIA } \\
\cline { 2 - 3 } & \text { v. 8, n. 14, p. 399-413, jul./dez. 2020. ISSN 2319-0566 }
\end{array}
$$


de Medicina, e as possíveis situações relacionadas às grávidas e puérperas existentes no âmbito hospitalar.

A demanda dos atendimentos se deu pelo fato de muitas gestantes estarem temerosas quanto à ida aos hospitais e aos demais locais físicos de serviço à saúde, uma vez que o risco de contaminação da COVID-19 é alto nesses ambientes. Além disso, a procura pelo serviço foi impulsionada através da divulgação do projeto pelo Instagram ${ }^{\circledR}$ dos voluntários e o telejornal regional.

Dentro desse cenário, verificou-se a correta disposição de muitas pacientes em acompanhar as mudanças no próprio corpo e como isso poderia afetar o filho, mobilizando-as a buscar entender e enfrentar os obstáculos do período gestacional. Dessa forma, também é de extrema importância a criação do vínculo entre a mulher e o profissional da saúde, o que pode proporcionar à gestante melhorias relacionadas à autoestima e à compreensão das modificações ocorridas nesse período (BONADIO et al., 1998).

\section{Conclusão}

Com base no que foi abordado, sabe-se que a pandemia da COVID-19 trouxe a necessidade de se buscar formas de atendimento seguro para a população em geral e, principalmente, de gestantes, uma vez que essas foram classificadas como grupo de risco. Nesse contexto, a implementação de um serviço de teleorientação permite uma consulta segura, a possibilidade de continuidade de assistência e uma redução da quantidade de atendimentos físicos, evitando superlotação e possíveis contaminações.

Desta forma, vale ressaltar que o projeto também contribuiu para o encaminhamento, quando necessário, das pacientes para os serviços de atenção à saúde adequados, de acordo com o grau de complexidade das queixas. Outrossim, o projeto promoveu a disseminação de informações embasadas cientificamente através do Instagram ${ }^{\circledR}$, abordando temas pertinentes à gestação e puerpério por meio de textos, vídeos e lives, dessa forma, contribuindo para a facilitação e ampliação do conhecimento ao público. Além disso, o projeto permitiu aos/às

$$
\begin{array}{l|l}
\multirow{2}{*}{\text { REVISTA }} & \text { EXTENSÃO \& CIDADANIA } \\
\cline { 2 - 3 } & \text { v. 8, n. 14, p. 399-413, jul./dez. 2020. ISSN 2319-0566 }
\end{array}
$$


alunos/as vivenciar e compartilhar experiências interprofissionais com profissionais da Enfermagem e Psicologia; possibilitou o contato com estudantes de outros períodos e proporcionou, ainda, a troca de diálogos com a população, contribuindo, dessa forma, para a formação discente.

\section{Referências}

AMORIM, M. M. R. et al. Clinical characteristics and risk factors for mortality in obstetric patients with severe COVID-19 in Brazil: a surveillance database analysis. BJOG: An International Journal of Obstetrics and Gynaecology, v. 127, n. 13, p. 1618-1626, 2020.

DORNA, C C. DOD/UEM participa do projeto "Teleorientação para Saúde Bucal em tempos de pandemia". 2020. Disponível em:

http://www.asc.uem.br/index.php?option=com_content\&view=article\&id=24844:dod-uemparticipa-do-projeto-teleorientacao-para-saude-bucal-em-tempos-depandemia\&catid=986\&Itemid=210. Acesso em: 5 dez. 2020.

FREITAS, D. DE et al. A emergência do novo coronavírus e a "lei de quarentena" no Brasil / The emergency of the new coronavirus and the "quarantine law" in Brazil. Revista Direito e Práxis, v. 0, n. 0, p. 1-38, 2020.

INSTITUTO PATRÍCIA GALVÃO (IPG). Mulheres Grávidas e Puérperas diante do Coronavírus. Online ONU Mulheres. 2020. Disponível em: https://assets-institucionalipg.sfo2.cdn.digitaloceanspaces.com/2020/10/INSTITUTOPATRICIAGALVAOLOCOMOT IVA_RelatorioGravidezeCovidVersaoFinal.pdf. Acesso em: 8 out. 2020.

LEVY, A. et al. ACE2 expression and activity are enhanced during pregnancy. American Journal of Physiology - Regulatory Integrative and Comparative Physiology, v. 295, n. 6, p. 1953-1962, 2008.

LIU, Y. et al. Aerodynamic analysis of SARS-CoV-2 in two Wuhan hospitals. Nature, v. 582, n. 7813, p. 557-560, 2020.

MENDES, E. Secretaria Municipal da Casa Civil. Diário Oficial Eletrônico do Município de Florianópolis. 2020. Disponível em: https://diarios.s3.amazonaws.com/DOM-FLNSC/2020/07/pdf/20200716_13.pdf?AWSAccessKeyId=AKIARMMD5JEAD4VJ344N\&Expir

$$
\begin{array}{c|l}
\multirow{2}{*}{\text { REVISTA }} & \text { EXTENSÃO \& CIDADANIA } \\
\cline { 2 - 3 } & \text { v. 8, n. 14, p. 399-413, jul./dez. 2020. ISSN 2319-0566 }
\end{array}
$$


es $=1607185037 \&$ Signature $=$ spNhpHrCphDgG1013mMF6M\%2BO9AM\%3D. Acesso em: 5 dez. 2020.

MENEZES, M. O. et al. Risk factors for adverse outcomes among pregnant and postpartum women with acute respiratory distress syndrome due to COVID-19 in Brazil. International Journal of Gynecology and Obstetrics, v. 151, n. 3, p. 415-423, 2020.

MINISTÉRIO DA SAÚDE (MS) (org.). COVID-19: painel coronavírus. Painel Coronavírus. 2020. Disponível em: https://covid.saude.gov.br/. Acesso em: 5 dez. 2020.

ROBERTON, T. et al. Early estimates of the indirect effects of the COVID-19 pandemic on maternal and child mortality in low-income and middle-income countries: a modelling study. The Lancet Global Health, v. 8, n. 7, p. e901-e908, 2020.

ROSENBERG, E. S. et al. Association of Treatment with Hydroxychloroquine or Azithromycin with In-Hospital Mortality in Patients with COVID-19 in New York State.

JAMA - Journal of the American Medical Association, v. 323, n. 24, p. 2493-2502, 2020.

ROYAL COLLEGE OF OBSTETRICIANS AND GYNECOLOGISTS (RCOG).

Coronavirus (COVID-19) Infection in Pregnancy. Guidelines [Internet], p.1-68, 2020.

Available from: https://www.rcog.org.uk/globalassets/documents/guidelines/2020-04-17coronavirus-covid-19-infection-in-pregnancy.pdf. Acesso em: 5 dez. 2020.

TAKEMOTO, M. L. S. et al. The tragedy of COVID-19 in Brazil: 124 maternal deaths and counting. International Journal of Gynecology and Obstetrics, v. 151, n. 1, p. 154-156, 2020.

TANG, K. et al. Erratum: Sexual and reproductive health (SRH): A key issue in the emergency response to the coronavirus disease (COVID- 19) outbreak (Reprod Health (2020) 17:59 DOI: 10.1186/s12978-020-0900-9). Reproductive Health, v. 17, n. 1, p. 17-19, 2020.

TAN, W. et al. A Novel Coronavirus from Patients with Pneumonia in China, 2019. New England Journal of Medicine, v. 382, n. 8, p. 727-733, 2020. World Health Organization.

TEIXEIRA, Marcionila. Serviço online inédito tira dúvidas de gestantes durante pandemia. 2020. Disponível em:

https://www.diariodepernambuco.com.br/noticia/vidaurbana/2020/04/servico-online-ineditotira-duvidas-de-gestantes-durante-pandemia.html. Acesso em: 5 dez. 2020.

WORLD HEALTH ORGANIZATION (WHO). WHO Coronavirus Disease (COVID-19). Dashboard. 2020. Disponível em: https://covid19.who.int/. Acesso em: 5 dez. 2020. 
ZAMBRANO, L. D. et al. Update: Characteristics of Symptomatic Women of Reproductive Age with Laboratory-Confirmed SARS-CoV-2 Infection by Pregnancy Status - United States, January 22-October 3, 2020. MMWR. Morbidity and Mortality Weekly Report, v. 69, n. 44, p. 1641-1647, 2020.

Recebido em: 19 de outubro de 2020.

Aceito em: 6 de dezembro de 2020 . 